\title{
Background phase correction in congenital heart disease: does reliability vary based on underlying disease type?
}

Sassan Hashemi ${ }^{1 *}$, Senthil Ramamurthy ${ }^{1}$, W James Parks ${ }^{1,2}$, Denver Sallee ${ }^{1,2}$, Tim Slesnick $^{1,2}$

From 19th Annual SCMR Scientific Sessions

Los Angeles, CA, USA. 27-30 January 2016

\section{Background}

Phase-contrast magnetic resonance (PC-MR) allows non-invasive calculation of vascular flow, peak velocities and shunts. The technique, however, has inherent limitations, one of which is background phase errors.
Various background phase correction (BPC) algorithms have been developed. The aim of this study is to apply various commercially available BPC algorithms in pediatric patients with a variety of disease types.

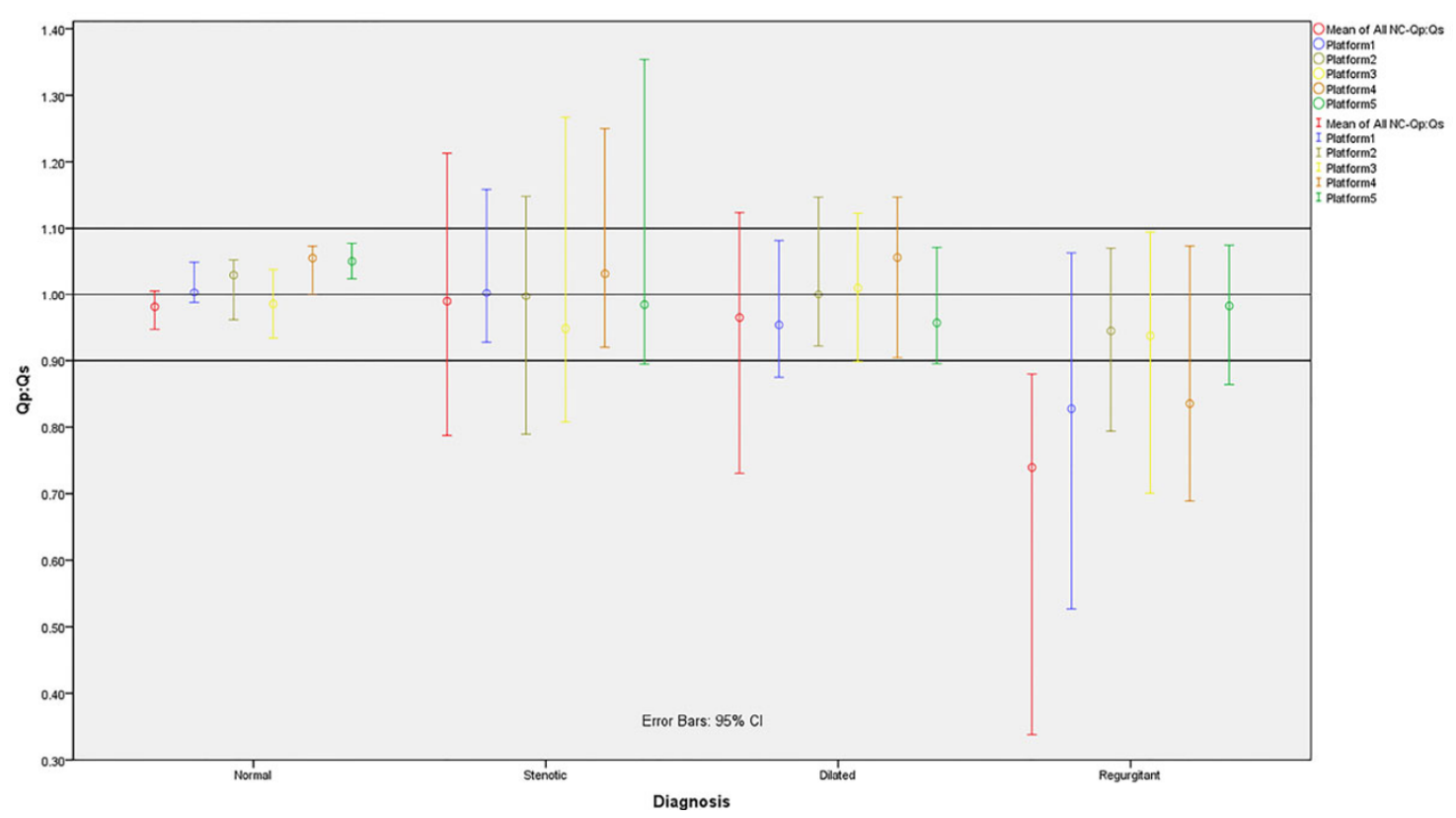

Figure 1 Distribution of Qp:Qs in different categories for all platforms. NC-Qp:Qs is the mean across all platforms. Data is represented as median and $95 \%$ confidence interval.

${ }^{1}$ Cardiovascular Imaging Research Core, Children's Healthcare of Atlanta,

Atlanta, GA, USA

Full list of author information is available at the end of the article

(0) 2016 Hashemi et al. This is an Open Access article distributed under the terms of the Creative Commons Attribution License (http:// creativecommons.org/licenses/by/4.0), which permits unrestricted use, distribution, and reproduction in any medium, provided the 


\begin{tabular}{|c|c|c|c|c|c|c|c|c|c|c|c|c|c|c|c|c|}
\hline & \multicolumn{4}{|c|}{ Normal } & \multicolumn{4}{|c|}{ Stenotic } & \multicolumn{4}{|c|}{ Dilated } & \multicolumn{4}{|c|}{ Regurgitant } \\
\hline Statistics & \begin{tabular}{|l} 
Median \\
(interquar \\
tile range)
\end{tabular} & $\begin{array}{l}\text { One } \\
\text { Sample } \\
\text { WSR for } \\
\text { Deviation } \\
\text { from } \\
\text { Qp:Qs=1: } 1^{1}\end{array}$ & $\begin{array}{l}\text { Related- } \\
\text { sample } \\
\text { WSR for } \\
\text { (NC) } \\
\text { vs. }(C)^{2}\end{array}$ & \begin{tabular}{|l|} 
Related- \\
sample \\
McNema \\
r for \\
(NC) \\
vs.(C) \\
(10\% \\
Offset)
\end{tabular} & \begin{tabular}{|l|} 
Median \\
(interquar \\
tile range)
\end{tabular} & $\begin{array}{l}\text { One } \\
\text { Sample } \\
\text { WSR for } \\
\text { Deviation } \\
\text { from } \\
\text { Qp:Qs=1:1 }\end{array}$ & $\begin{array}{l}\text { Related- } \\
\text { sample } \\
\text { WSR for } \\
\text { (NC) } \\
\text { vs.(C) }\end{array}$ & $\begin{array}{l}\text { Related- } \\
\text { sample } \\
\text { McNema } \\
\text { r for (NC) } \\
\text { vs.(C) } \\
(10 \% \\
\text { Offset) }\end{array}$ & $\begin{array}{l}\text { Median } \\
\text { (interquar } \\
\text { tile range) }\end{array}$ & $\mid \begin{array}{l}\text { One } \\
\text { Sample } \\
\text { WSR for } \\
\text { Deviation } \\
\text { from } \\
\text { Qp:Qs=1:1 }\end{array}$ & \begin{tabular}{|l} 
Related- \\
sample \\
WSR for \\
(NC) \\
vs.(C)
\end{tabular} & $\begin{array}{l}\text { Related- } \\
\text { sample } \\
\text { McNema } \\
\text { r for (NC) } \\
\text { vs.(C) } \\
(10 \% \\
\text { Offset) }\end{array}$ & $\begin{array}{l}\text { Median } \\
\text { (interquar } \\
\text { tile range) }\end{array}$ & $\begin{array}{l}\text { One Sample } \\
\text { WSR for } \\
\text { Deviation } \\
\text { from } \\
\text { Qp:Qs=1:1 }\end{array}$ & $\begin{array}{l}\text { Related- } \\
\text { sample } \\
\text { WSR for } \\
\text { (NC) vs.(C) }\end{array}$ & $\begin{array}{l}\text { Related- } \\
\text { sample } \\
\text { McNema } \\
\text { r for (NC) } \\
\text { vs.(C) } \\
\text { (10\% } \\
\text { Offset) }\end{array}$ \\
\hline $\begin{array}{l}\text { Platform } 1 \\
\text { (NC) }\end{array}$ & $0.97(0.06)$ & $P=0.002$ & \multirow{2}{*}{$P<0.001$} & \multirow{2}{*}{$P=1.0$} & $0.99(0.23)$ & $P=0.58$ & \multirow{2}{*}{$P=0.09$} & \multirow{2}{*}{$P=0.5$} & $0.93(0.27)$ & $P=0.047$ & \multirow{2}{*}{$P=0.03$} & \multirow{2}{*}{$P=1.0$} & $0.74(0.47)$ & $P=0.005$ & \multirow{2}{*}{$P=0.02$} & \multirow{2}{*}{$P=0.50$} \\
\hline $\begin{array}{l}\text { Platform } 1 \\
\text { (C) }\end{array}$ & $1.00(0.07)$ & $P=0.53$ & & & $1.00(0.15)$ & $P=0.72$ & & & $0.95(0.18)$ & $P=0.33$ & & & $0.83(0.43)$ & $P=0.06$ & & \\
\hline $\begin{array}{l}\text { Platform 2 } \\
\text { (NC) }\end{array}$ & $0.97(0.1)$ & $P=0.04$ & \multirow{2}{*}{$\mathrm{P}=0.001$} & \multirow{2}{*}{$P=0.45$} & $0.94(0.32)$ & $P=0.45$ & \multirow{2}{*}{$P=0.8$} & \multirow{2}{*}{$P=1.0$} & $0.97(0.28)$ & $P=0.24$ & \multirow{2}{*}{$P=0.24$} & \multirow{2}{*}{$P=0.25$} & $0.73(0.35)$ & $P=0.005$ & \multirow{2}{*}{$P=0.005$} & \multirow{2}{*}{$P=0.06$} \\
\hline $\begin{array}{l}\text { Platform } 2 \\
\text { (C) } \\
\end{array}$ & $1.03(0.1)$ & $P=0.33$ & & & $1.00(0.29)$ & $\mathrm{P}=0.88$ & & & $1.00(0.14)$ & $P=0.96$ & & & $0.94(0.25)$ & $P=0.14$ & & \\
\hline $\begin{array}{l}\text { Platform } 3 \\
\text { (NC) } \\
\end{array}$ & $0.97(0.1)$ & $\mathrm{P}=0.04$ & \multirow{2}{*}{$P=0.09$} & \multirow{2}{*}{$P=1.0$} & $0.94(0.32)$ & $P=0.45$ & \multirow{2}{*}{$P=0.24$} & \multirow{2}{*}{$P=1.0$} & $0.97(0.28)$ & $P=0.24$ & \multirow{2}{*}{$P=0.03$} & \multirow{2}{*}{$P=0.63$} & $0.73(0.35)$ & $P=0.005$ & \multirow{2}{*}{$P=0.005$} & \multirow{2}{*}{$P=0.25$} \\
\hline $\begin{array}{l}\text { Platform } 3 \\
\text { (C) }\end{array}$ & $0.99(0.13)$ & $P=0.31$ & & & $0.95(0.35)$ & $P=0.96$ & & & $1.01(0.13)$ & $P=0.72$ & & & $0.94(0.34)$ & $P=0.11$ & & \\
\hline $\begin{array}{l}\text { Platform } 4 \\
\text { (NC) } \\
\end{array}$ & $0.98(0.08)$ & $P=0.35$ & \multirow{2}{*}{$P<0.001$} & \multirow{2}{*}{$P=1.0$} & $1.00(0.29)$ & $P=0.96$ & \multirow{2}{*}{$P=0.005$} & \multirow{2}{*}{$P=0.5$} & $0.98(0.29)$ & $P=0.17$ & \multirow{2}{*}{$\mathrm{P}=0.02$} & \multirow{2}{*}{$P=1.0$} & $0.73(0.38)$ & $P=0.005$ & $P=0,02$ & $P=0.50$ \\
\hline $\begin{array}{l}\text { Platform } 4 \\
\text { (C) }\end{array}$ & $1.05(0.09)$ & $P=0.04$ & & & $1.03(0.22)$ & $\mathrm{P}=0.22$ & & & $1.06(0.22)$ & $P=0.33$ & & & $0.83(0.35)$ & $P=0.07$ & $p=0.02$ & $=0.50$ \\
\hline $\begin{array}{l}\text { Platform } 5 \\
\text { (NC) } \\
\end{array}$ & $0.99(0.1)$ & $\mathrm{P}=0.11$ & $P<0,001$ & $P=0.25$ & $1.05(0.32)$ & $\mathrm{P}=0.58$ & $P=45$ & $P=1.0$ & $0.96(0.22)$ & $P=0.14$ & $P=0.45$ & $P=0,25$ & $0.74(0.47)$ & $P=0.005$ & $P=0.007$ & $P=0.22$ \\
\hline $\begin{array}{l}\text { Platform } 5 \\
\text { (c) }\end{array}$ & $1.05(0.07)$ & $\mathrm{P}=0.02$ & $p<0.001$ & $P=0.23$ & $0.98(0.36)$ & $\mathrm{P}=0.56$ & & & & $P=0.45$ & & & & $P=0.51$ & $P=0.007$ & $P=0.22$ \\
\hline $\begin{array}{l}\text { Figure } 2 \\
\text { equals } 1 \text { : } \\
\text { hypothes } \\
\text { acceptab } \\
\text { correctio }\end{array}$ & $\begin{array}{l}2 \text { (C): Co } \\
: 1(P<0 . \\
\text { sis). 3. Nu } \\
\text { le in (NC } \\
\text { n algorith }\end{array}$ & $\begin{array}{l}\text { orrected } \\
.05 \text { rejec } \\
\text { ull Hypot } \\
\text { c) vs. (C) } \\
\text { hms in }\end{array}$ & $\begin{array}{l}\text { Qp:Qs, ( } \\
\text { null hyp } \\
\text { hesis: The } \\
0.9: 1.0 \leq \\
\text { same po }\end{array}$ & $\begin{array}{l}\text { (NC): } \mathbf{N} \\
\text { pothesis } \\
\text { ere is no } \\
\text { Qp:Qs } \\
\text { ost-proce }\end{array}$ & $\begin{array}{l}\text { Jon-corre } \\
\text { s). } 2 \text {. Null } \\
\text { o significar } \\
\leq 1.1: 1.0 \\
\text { essing par }\end{array}$ & $\begin{array}{l}\text { ected } \\
\text { Hypotl } \\
\text { ant diffe } \\
\text { was se } \\
\text { ackage, }\end{array}$ & $\begin{array}{l}\text { p:Qs, } \\
\text { esis: Th } \\
\text { rence } \\
\text { as clin } \\
\text { hus sh }\end{array}$ & $\begin{array}{l}\text { ISR: Wilc } \\
\text { median } \\
\text { atween di } \\
\text { cally acce } \\
\text { ring the s }\end{array}$ & $\begin{array}{l}\text { Icoxon Si } \\
\text { of distrib } \\
\text { distributio } \\
\text { eptable). } \\
\text { same nor }\end{array}$ & $\begin{array}{l}\text { igned } R \mathbf{~} \\
\text { bution be } \\
\text { on of dich } \\
\text { 4. Platforn } \\
\text { n-correcte }\end{array}$ & $\begin{array}{l}\text { lank } \\
\text { etwee } \\
\text { hoton } \\
\text { m } 2 \\
\text { ted d }\end{array}$ & $\begin{array}{l}\text { st. 1. Nu } \\
\text { (NC) vs } \\
\text { zed Qp:Q } \\
\text { d } 3 \text { are t }\end{array}$ & $\begin{array}{l}\text { ull hypoth } \\
\text { (C) equals } \\
\text { 2s into clin } \\
\text { two differe }\end{array}$ & $\begin{array}{l}\text { hesis: The } \\
\text { Is " } 0 \text { " }(P<0 \\
\text { nically acce } \\
\text { ent backgr }\end{array}$ & $\begin{array}{l}\text { median } \\
0.05 \text { rejec } \\
\text { eptable ar } \\
\text { round ph }\end{array}$ & $\begin{array}{l}\text { of Qp:Q } \\
\text { cts null } \\
\text { and not } \\
\text { hase }\end{array}$ \\
\hline
\end{tabular}

\section{Methods}

Retrospectively, we analyzed patients in 4 categories: normal anatomy, stenotic aortic valve, dilated aortic root, and regurgitant pulmonary valve. All patients had PC-MR data obtained on a $1.5 \mathrm{~T}$ magnet (Siemens MAGNETOM Aera) using a product sequence optimized for pediatric patients with free breathing techniques, multiple signal averages, and the vessel of interest placed at isocenter. We excluded patients with intracardiac shunting, arrhythmias or prosthetic valves. We calculated the aortic to pulmonary flow ratio (Qp:Qs) on 5 different analysis platforms both with and without BPC. Three platforms utilize a full-field stationary tissue fit and two others use a region of interest (ROI) for BPC, which was placed in stationary tissue as close to the vessel of interest as possible. One expert reader performed all vessel segmentations, ensuring all segmentations on different platforms used similar technique. Qp:Qs between 0.9 - 1.1 was defined as clinically acceptable.

\section{Results}

Fifty patients ( $76 \%$ males, mean age $=13 \pm 5$ years $)$ were analyzed (20 normal, 10 each from the other groups). The intraclass correlation coefficient for intra-observer reliability was 0.99. Distributions of Qp:Qs for different disease categories, before and after BPC, on all platforms are summarized in Table 1 and Figure 1. Non-corrected (NC) Qp:Qs in normal patients are distributed in the clinically acceptable range. The worst underestimation of Qp:Qs occurred in the regurgitant group, and BPC was significantly beneficial for these patients, with platform 5 being the most efficient. In the dilated aorta group, only the outlier Qp:Qs measurements benefitted from BPC, but the distribution of data were mostly in the acceptable range. BPC could not compensate for outlier measurements in the stenotic aortic valve group, though the medians of distribution did not deviate from 1.0.

\section{Conclusions}

Non-corrected phase contrast values vary in clinical accuracy based on underlying disease type, and there are significant differences between various vendors' BPC algorithm efficiencies at resolving these discrepancies. These effects were most pronounced in pediatric patients with regurgitant lesions.

\section{Authors' details}

${ }^{1}$ Cardiovascular Imaging Research Core, Children's Healthcare of Atlanta, Atlanta, GA, USA. ${ }^{2}$ Pediatrics, Emory University School of Medicine, Atlanta, GA, USA. 
doi:10.1186/1532-429X-18-S1-094

Cite this article as: Hashemi et al:: Background phase correction in congenital heart disease: does reliability vary based on underlying disease type? Journal of Cardiovascular Magnetic Resonance 2016 18(Suppl 1):094.

Submit your next manuscript to BioMed Central and take full advantage of:

- Convenient online submission

- Thorough peer review

- No space constraints or color figure charges

- Immediate publication on acceptance

- Inclusion in PubMed, CAS, Scopus and Google Scholar

- Research which is freely available for redistribution

Submit your manuscript at www.biomedcentral.com/submit 